\title{
On membrane diffusion with near-equilibrium reaction
}

\author{
J. D. GODDARD, J. S. SCHULTZ and R. J. BASSETT \\ University of Michigan, Department of Chemical and Metallurgical Engineering, Ann Arbor, \\ Mich. 48104, U.S.A.
}

(First received 10 April 1969; accepted 24 September 1969)

\begin{abstract}
An analysis is presented of the "facilitated" transport problem, involving steady diffusion through a finite membrane, accompanied by rapid and reversible, homogeneous chemical reaction. For the limit of infinitely fast reactions, the method of matched asymptotic expansions is used to analyze the asymptotic structure of the diffusion field, which is shown to consist of an "equilibrium core," together with "boundary-layer" reaction zones at the membrane boundaries. Asymptotic expansions are derived, which would in principle allow one to compute both the first- and second-order effects of departure from reaction equilibrium on the net rates of diffusion mass transfer. Several examples are considered, one of which is a model of oxygen diffusion through hemoglobin solutions, and some comparisons with analytical and numerical solutions are given.
\end{abstract}

\section{INTRODUCTION}

IT IS GENERALLY acknowledged that chemical reactions can often have an important influence on the rates of diffusional transport. While there has been a long-standing interest, particularly on the part of chemists and chemical engineers, in the effects of homogeneous chemical reaction on rates of transport processes, many recent studies in this area have been specifically oriented towards biological systems and the reactive "enhancement", or "facilitation", of diffusion as it occurs in membranes, cells, or liquid films. An example which figures prominently here is, quite naturally, the hemoglobinoxygen system $[9,12]$.

Since, more generally, there is known to exist a wide variety of both heat- and mass-transport processes in which the effects of chemical reaction may have practically interesting and, indeed, beneficial consequences, the important question arises as to the maximum effect that can be realized in a particular process. In the usual cases, one expects that this maximum will correspond to the theoretical limit of infinitely fast reaction, which in part accounts for the rather extensive literature dealing with the effects of rapid homogeneous reaction on diffusion $[4,5$, $13,15,18,20,21]$. For example, in one such work, directed mainly towards application to the classical "film" theories of convective mass transfer. Olander[15] has presented a rather extensive analysis of one-dimensional diffusion in the presence of homogeneous reactions, which were considered to be so rapid as to achieve the limit of reaction equilibrium everywhere in the diffusion field. Also, Hirschfelder [13] has given an analysis of heat transfer in the presence of rapid homogeneous, as well as heterogeneous, reactions.

In the present work we are concerned with a related problem, which deals with the steadystate diffusion of a chemical species through a medium of finite dimensions in which it reacts chemically with one or several "non-transferable" species that remain permanently entrapped within the medium. In particular, the present analysis will deal mainly with one-dimensional diffusion through films or membranes, and, to be consistent with our assumption of a steady state and the permanence of certain reactants, we will consider only reversible chemical reactions. This immediately distinguishes the type of analysis to be given here from those which involve possibly irreversible reactions, for example, such as the analysis of Olander [15] cited above.

We are particularly concerned here with the 
above-mentioned extreme of rapid chemical reaction, and the analysis employed will hopefully prove to be useful in a number of important problems involving transport through diffusional barriers. One of the principal objectives of this work is to elucidate the asymptotic structure of the diffusion field in the limit of rapid, "nearequilibrium" reaction. In this regard, one should take care to distinguish between the concept of "near-equilibrium" reaction, as it is employed here, and that of equilibrium in the thermodynamic sense, as it applies to systems in which all driving forces or "affinity" gradients are small $[6,8,16]$.

It would appear that, aside from the works on diffusion with reaction, such as that of Friedlander and Keller[8], which are based on the latter concept of equilibrium and are therefore valid only in the limit of small diffusional driving force, most of the previous theoretical treatments rest on some rather intuitive, ad hoc assumptions as to the precise asymptotic nature of the "near-equilibrium" state as it applies to rapid reactions. While the work of Hirschfelder [13] on the related heat-transfer problem clearly anticipates the "boundary-layer" structure of such systems, it does not appear to provide a generally applicable mathematical analysis. As will be shown here, the rather recently developed "method of matched asymptotic expansions" which has found numerous applications to other transport phenomena (and the rudiments of which are given in a recent, very readable account by Acrivos [1]) affords us with a natural analysis of this type of system, which appears to have been suggested in a previous work of Murray [14] on a related problem. In addition to providing a satisfactorily rigorous definition of the near-equilibrium state, this technique also allows a rather straight-forward computation of terms to account for departure from this state.

To illustrate the application of this method, a system has been chosen which is simple enough to allow some physical insight but which, at the same time, will permit the development of a formal method of attack that should be applicable to more complicated problems.

\section{ONE-DIMENSIONAL DIFFUSION WITH} RAPID ISOTHERMAL REACTION

We now consider the steady molecular diffusion of a chemical species A through a membrane (or solution) in the form of a planar slab of thickness $L$ located, say, in a region $0<x<L$. In the interior of the membrane the species $A$ undergoes a reversible chemical reaction with a second diffusing species $\mathbf{B}$, say,

$$
a \mathbf{A}+b \mathbf{B} \rightleftarrows \mathbf{C}
$$

where C is a diffusing "product" or "carrier" species. To facilitate the discussion here, we postulate uniform temperature and we adopt the elementary form of "Fick's law", for dilute solutions with no bulk flow, no diffusive coupling, and with constant diffusivities. In this case, the transport equations for one-dimensional diffusion with chemical reaction assume their wellknown form:

$$
\frac{D_{A}}{a} \frac{\mathrm{d}^{2} C_{A}}{\mathrm{~d} x^{2}}=\frac{D_{B}}{b} \frac{\mathrm{d}^{2} C_{B}}{\mathrm{~d} x^{2}}=-D_{C} \frac{\mathrm{d}^{2} C_{C}}{\mathrm{~d} x^{2}}=r
$$

where $a$ and $b$ are the stoichiometric ratios of (2.1), the C's and $D$ 's denote, respectively, the molar concentrations and diffusivities of the various species, and, finally, $r$ is the reaction rate, expressed as the molar-volumetric generation rate of the product $\mathbf{C}$. For the present purposes, the kinetic function $r=r\left(C_{A}, C_{B}, C_{C}\right)$ can be treated as an aribtrary function of the concentrations, with the proviso that it admits of a family of equilibrium" concentrations, $C^{(0)}$, such that

$$
r\left(C_{A}{ }^{(0)}, C_{B}{ }^{(0)}, C_{C}{ }^{(0)}\right)=0 .
$$

Because of the applications contemplated here, we presume that only the species $A$ is transferred, i.e. has a non-zero net flux, through the membrane, whereas the species $B$ and $C$ are considered to be "non-transferable". Accordingly, at the faces of the membrane $x=0, L$, which represent phase boundaries with the surroundings, the concentration of $\mathbf{A}$ is assumed to 
be known while the diffusive fluxes of $\mathbf{B}$ and $\mathbf{C}$ must both vanish. Therefore, we have as boundary conditions that

$$
C_{A}=\bar{C}, \frac{\mathrm{d} C_{B}}{\mathrm{~d} x}=\frac{\mathrm{d} C_{C}}{\mathrm{~d} x}=0, \quad \text { at } x=0,
$$

and

$$
C_{A}=\underline{C}, \frac{\mathrm{d} C_{B}}{\mathrm{~d} x}=\frac{\mathrm{d} C_{C}}{\mathrm{~d} x}=0, \quad \text { at } x=L,
$$

where $\bar{C}$ and $\underline{C}$ are specified constants of the problem.

A full description of the diffusion process requires of course a solution to (2.2) subject to the conditions of (2.3). To complete the set of governing equations, we note that a simple integration of (2.2) yields

$$
\begin{aligned}
\int_{0}^{L} r \mathrm{~d} x=-\left.D_{C} \frac{\mathrm{d} C_{C}}{\mathrm{~d} x}\right|_{0} ^{L}= & \left.\frac{D_{B}}{b} \frac{\mathrm{d} C_{B}}{\mathrm{~d} x}\right|_{0} ^{L} \\
& =\left.\frac{D_{A}}{a} \frac{\mathrm{d} C_{A}}{\mathrm{~d} x}\right|_{0} ^{L}=0,
\end{aligned}
$$

the last equality resulting from the zero-flux conditions of (2.3). Now, from (2.4) it follows that the net rate of reaction in the membrane is zero, and of course that the diffusive flux of the species $^{-1} \mathbf{A}$ is the same at either boundary. Moreover, it can be seen by (2.4) that only three of the four flux conditions in (2.3) are independent. As a consequence, it is necessary (for the sake of uniqueness) to specify a further restriction on the solution to (2.2), which on physical grounds we take to be the stoichiometric constraint

$$
\int_{0}^{L}\left(C_{C}+C_{B} / b\right) \mathrm{d} x=L C_{0}
$$

where $C_{0}$ is a specified molar concentration. This fixes the total amount of the non-transferable species $\mathbf{B}$ and $\mathbf{C}$ present in the membrane which is expressed here as molar equivalents of species C.

With the problem statement now complete, we can qualitatively anticipate as its solution a set of concentration and reaction-rate profiles such as those shown in Fig. 1, which refers to a situation

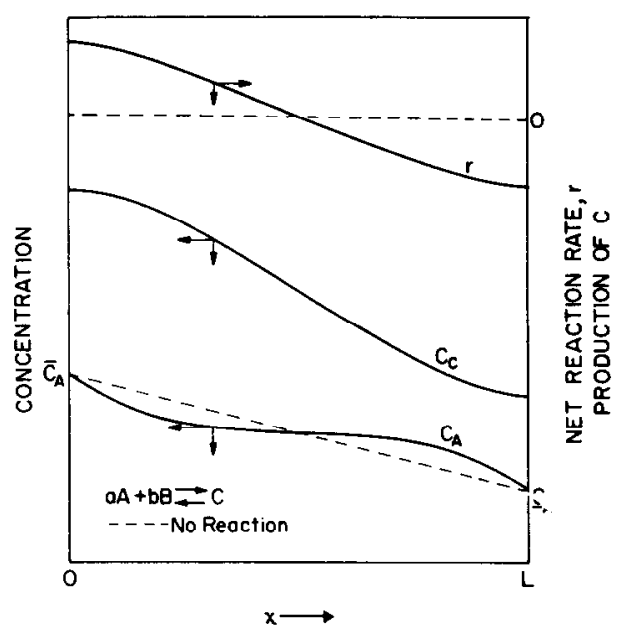

Fig. 1. Qualitative sketch of concentration and reaction rate profiles ( $C_{B}$ not shown).

where there is presumably a net flux of $\mathbf{A}$ from left $(x=0)$ to right $(x=L)$, corresponding to a "driving force" $\bar{C}-\underline{C}>0$. Thus, at the left-hand boundary, the species $A$ is absorbed at the concentration level $\bar{C}$, and the reaction (2.1) proceeds to the right. Then, after diffusing in both the free (as A) and combined forms (as the "carrier" C), A is desorbed at the right-hand boundary, where conversely (2.1) proceeds to the left.

In the final analysis, the quantity desired is the net molar flux of $\mathbf{A}$, given by

$$
N_{A}=-D_{A}\left(\frac{\mathrm{d} C_{A}}{\mathrm{~d} x}\right)_{x=0} \equiv-D_{A}\left(\frac{\mathrm{d} C_{A}}{\mathrm{~d} x}\right)_{x=L} .
$$

We are mainly concerned in the present work with the asymptotic flux in the limiting case where reaction rates become extremely large compared to diffusion rates. To formulate this condition in terms of the parameters of our problem, and also to facilitate the notation of the following analysis, it will be convenient to express Eqs. (2.2-2.4) in the dimensionless form

$$
\epsilon^{2} \frac{\mathrm{d}^{2} \psi_{i}}{\mathrm{~d} y^{2}}-\mu_{i} \phi=0,(i=1,2,3)
$$


with

$$
\begin{array}{ll}
\psi_{1}=\bar{\psi}, \frac{\mathrm{d} \psi_{2}}{\mathrm{~d} y}=\frac{\mathrm{d} \psi_{3}}{\mathrm{~d} y}=0, & \text { at } y=0, \\
\psi_{1}=\psi, \frac{\mathrm{d} \psi_{2}}{\mathrm{~d} y}=\frac{\mathrm{d} \psi_{3}}{\mathrm{~d} y}=0, & \text { at } y=1,
\end{array}
$$

and

$$
\int_{0}^{1}\left(\psi_{2}+\psi_{3}\right) \mathrm{d} y=1
$$

where

$y=x / L, \psi_{1}=C_{A} / a C_{0}, \psi_{2}=C_{B} / b C_{0}, \psi_{3}=C_{C} / C_{0}$

$\epsilon^{2}=\frac{D^{*} C_{0}}{\mathrm{~L}^{2} r^{*}}, \mu_{1}=D^{*} / D_{A}, \mu_{2}=D^{*} / D_{B}$,

$\mu_{3}=-D^{*} / D_{C}, \quad \bar{\psi}=\bar{C} / a C_{0}, \underline{\psi}=\underline{C} / a C_{0}$,

and

$$
\phi=\phi\left(\psi_{1}, \psi_{2}, \psi_{3}\right)=r\left(C_{A}, C_{B}, C_{C}\right) / r^{*} .
$$

Here, $r^{*}$ is a characteristic magnitude of the reaction rate $r$ (its value, say, at some characteristic, but non-equilibrium set of concentrations) and $D^{*}$ is some typical diffusivity (for example, any one of the individual diffusivities $\left.D_{A}, \ldots ..\right)$.

The parameter $\epsilon$, defined by (2.9), provides us with a measure of the importance of diffusion relative to reaction, and thus we seek the asymptotic solution to (2.6), (2.7), and (2.8) for $\epsilon \rightarrow 0$, with all other parameters fixed. It can be recognized immediately from the form of (2.6) that this problem should almost certainly involve a "singular-perturbation", or non-uniformily valid, asymptotic solution. Indeed, if the term in $\epsilon^{2}$ is neglected from (2.6) we obtain, in the first approximation, a condition of reaction equilibrium everywhere:

$$
\phi\left(\psi_{1}, \psi_{2}, \psi_{3}\right) \equiv 0,
$$

which in general will not admit solutions $\psi_{i}$ that satisfy all the boundary conditions of (2.7) and (2.8).

On the basis of these observations, and by analogy with the structure of other, markedly similar physical systems, we are quite naturally led to anticipate the occurrence of two types of asymptotic solution to (2.6), each with different but overlapping regions of validity. Specifically, we postulate the existence of an "equilibrium core", $0(\epsilon)<y<1-0(\epsilon)$, where the condition of (2.10) is satisfied identically for $\epsilon \rightarrow 0$, together with "reaction boundary layers" in the neighborhoods of the boundaries, $0 \leqslant y<0(\epsilon), 1-0(\epsilon)$ $<y \leqslant 1$, where both reaction and diffusion rates are extremely rapid. In these latter regions, both the diffusion and reaction terms in (2.6) will presumably be of the same order of magnitude. (We might note here that our terminology "equilibrium core" follows the usage, "inviscid core", of Batchelor [3] for an analogous problem in fluid mechanics. The one-dimensional problem we consider here can be regarded, of course, as a special case of the more general mass-transfer problem in two or three spatial dimensions).

With the preceding remarks in mind, we turn now to the actual formulation of suitable asymptotic expansions for the present problem which, in addition to yielding the limiting form of mass transfer for $\epsilon \rightarrow 0$, will permit a systematic derivation of correction terms, for small but non-zero $\epsilon$.

\subsection{Formulation of the expansions}

Before writing down expansions in $\epsilon$ for the concentration variables $\psi_{i}$ of (2.9), it is expedient to derive certain integral relations from (2.6) which hold for any $\epsilon$ and which consequently involve small- $\epsilon$ expansions. The relations in question, which we shall call kinetic constraints, can be obtained simply by multiplying the differential equation for $\psi_{i}$ in (2.6) by $\mu_{j}$, forming the antisymmetric difference to eliminate the term in $\phi$, and integrating twice, which yields

$$
\mu_{i} \psi_{j}-\mu_{j} \psi_{i}=\alpha_{i j} \mathrm{y}+\beta_{i j}
$$

where the constants of integration $\alpha_{i j}, \beta_{i j}$, antisymmetric in the indices $i, j$, both satisfy equations of the form

$$
\alpha_{i j}=-\alpha_{j i}, \mu_{k} \alpha_{i j}=\mu_{i} \alpha_{k j}+\mu_{j} \alpha_{i k} \text {, for } i, j, k=1,2,3
$$


For the number of species considered here there are, generally speaking, only three independent members in each of the sets $\alpha$ and $\beta$ but, owing to the boundary conditions (2.7), we must have for the $\alpha$ 's that

$$
\alpha_{23}=-\alpha_{32}=\mathbf{0},
$$

which leaves us, then, with five parameters (e.g. $\left.\alpha_{12}, \alpha_{13}, \beta_{12}, \beta_{13}, \beta_{23}\right)$ of which only three are independent.

One will observe that, by means of the integral relations $(2.11)$, we could in principle eliminate any two of the functions $\psi_{i}$ by expressing them in terms of the remaining function, in combination with the above constants of integration. This leads, however, to a rather cumbersome, unsymmetric formulation of the expansion problem and thus will not be done at this stage. Nevertheless. we shall make use of the relations (2.11)-(2.13), and hence we emphasize that the constants in (2.11), when regarded as quantities to be determined, will depend on the parameter $\epsilon$. In fact, the quantity of interest here the mass flux of $\mathbf{A}$, can be expressed in terms of a dimensionless flux ratio $S$ (a Sherwood number), in the alternate forms

$$
\begin{aligned}
S & \equiv \frac{L N_{A}}{D_{A}(\bar{C}-C)}=-\left(\frac{1}{\bar{\psi}-\underline{\psi}}\right) \frac{\mathrm{d} \psi_{1}(0)}{\mathrm{d} y} \\
& =\frac{\alpha_{12}}{\mu_{2}(\bar{\psi}-\psi)}=\frac{\mu_{1}}{\mu_{2}}\left[\frac{\psi_{2}(1)-\psi_{2}(0)}{\bar{\psi}-\underline{\psi}}\right]+1 \\
& =\frac{\alpha_{13}}{\mu_{3}(\bar{\psi}-\underline{\psi})}=-\frac{\mu_{1}}{\mu_{3}}\left[\frac{\psi_{3}(0)-\psi_{3}(1)}{\bar{\psi}-\underline{\psi}}\right]+1,
\end{aligned}
$$

which follow readily from the relations given above. The non-zero $\alpha$ 's are thus directly related to the desired flux and also to the boundary values on the non-transferred species. This stated, we consider the expansion problem beginning with:

The core expansions. In the equilibrium core we postulate for the concentration fields a set of power-series expansion in $\epsilon$ of the type

$$
\psi_{i}=\Psi_{i}^{(0)}+\epsilon \Psi_{i}^{(1)}(y)+\epsilon^{2} \Psi_{i}^{(2)}(y)+0\left(\epsilon^{3}\right)
$$

where the $\Psi_{i}^{(p)}(p=0,1,2, \ldots, i=1,2,3)$ are independent of $\epsilon$. Taken together, (2.11) and (2.15) imply expansions such as

$$
\alpha_{i j}=\alpha_{i j}^{(0)}+\epsilon \alpha_{i j}^{(1)}+\epsilon^{2} \alpha_{i j}^{(2)}+0\left(\epsilon^{3}\right)
$$

for the non-zero $\alpha$ 's and $\beta$ 's of (2.12). Then, with the assumption of a Taylor-series expansion for the kinetic function $\phi\left(\psi_{1}, \psi_{2}, \psi_{3}\right)$ of $(2.9)$ in its arguments $\psi_{i}$, we obtain by means of (2.15) an expansion for $\phi$ of the form:

$$
\begin{aligned}
\phi= & \Phi+\epsilon \Phi^{j} \Psi_{j}^{(1)}+\epsilon^{2}\left[\Phi^{j} \Psi_{j}^{(2)}+\Phi^{j k} \Psi_{j}^{(1)} \Psi_{j}^{(1)}\right] \\
& +0\left(\epsilon^{3}\right),
\end{aligned}
$$

where

$\Phi=(\phi)_{\Psi^{(0)}}, \Phi^{j}=\left(\frac{\partial \phi}{\partial \psi_{j}}\right)_{\Psi^{(0)}}, \Phi^{j k}=\frac{1}{2 !}\left(\frac{\partial^{2} \phi}{\partial \psi_{j} \partial \psi_{k}}\right)_{\Psi^{(0)}}$

are the values of the function $\phi$ and its higher partial derivatives evaluated at $\psi_{i}=\Psi_{i}^{\left({ }^{(0)}\right.}$, which of course are the zeroth-order of equilibrium terms in (2.15). Also, for the sake of brevity, we have adopted, here, as in the following analysis, the "tensorial" summation convention for the derivatives of $\phi$ in (2.17) (whereby a term containing a repeated index, that appears as both superscript and subscript, indicates the sum of such terms over the entire range of indicial values, which here are $i, j, k, \ldots=1,2,3)$.

By the usual logic of such analyses, the set (2.6), (2.15), (2.16), and (2.17) leads to the following equations for the perturbation coefficients $\Psi_{i}^{(p)}, \alpha_{i j}^{(p)}$ and $\beta_{i j}^{(p)}$ :

$$
\begin{array}{r}
\Phi \equiv \phi\left(\Psi_{1}^{(0)}, \Psi_{2}^{(0)}, \Psi_{3}{ }^{(0)}\right) \equiv 0 \\
\Phi^{h} \Psi_{k}^{(1)}=0 \\
\mu_{i} \Phi^{k} \Psi_{k}^{(2)}=\left[\frac{\mathrm{d}^{2} \Psi_{i}^{(0)}}{\mathrm{d} y^{2}}-\mu_{i} \Phi^{j k} \Psi_{j}^{(1)} \Psi_{k}^{(1)}\right]
\end{array}
$$

with kinetic constraints,

$$
\mu_{i} \Psi_{j}^{(p)}-\mu_{j} \Psi_{i}^{(p)}=\alpha_{i j}^{(p)} y+\beta_{i j}^{(p)},
$$

for $i, j, \ldots=1,2,3, p=0,1,2, \ldots$, where the $\alpha^{(p)}$ and $\beta^{(p)}$ all satisfy relations like (2.12) and (2.13).

Equations (2.18) and (2.21) $(p=0)$ govern the 
equilibrium concentrations $\Psi_{i}^{\left({ }^{(1)}\right.}$ in the core, and the remaining relations are linear algebraic equations for the successively higher-order correction terms. The set of Eqs. (2.18)-(2.21) is not complete and, within the framework of the present treatment, the complete solution for the core function can be arrived at only by matching with corresponding terms in:

The boundary-layer expansions. We first treat the left-hand boundary, $y=0$, and, by analogy with other such boundary-layer problems, we postulate expansions of the form

$\psi_{i}=\bar{\psi}_{i}^{(0)}(\bar{y})+\epsilon \bar{\psi}_{i}^{(1)}(\bar{y})+\epsilon^{2} \bar{\psi}_{i}^{(2)}(\bar{y})+0\left(\epsilon^{3}\right)$

$(i=1,2,3)$ for the boundary-layer region, where the "strained" coordinate

$$
\bar{y}=y / \epsilon
$$

is $0(1)$. The functions $\bar{\psi}_{i}^{(p)}(\bar{y})$, which are independent of $\epsilon$, must be chosen to satisfy (2.6) as well as the conditions at $y=0$ in (2.7).

By a development parallel to that given above, we then obtain readily the following differential equations and boundary conditions from (2.6) and (2.7):

$$
\begin{gathered}
\frac{\mathrm{d}^{2} \bar{\psi}_{i}^{(0)}}{\mathrm{d} \bar{y}^{2}}-\mu_{i} \bar{\phi}=0, \\
\frac{\mathrm{d}^{2} \bar{\psi}_{i}^{(1)}}{\mathrm{d} \bar{y}^{2}}-\mu_{i} \bar{\phi}^{k} \bar{\psi}_{k}^{(1)}=0, \\
\frac{\mathrm{d}^{2} \bar{\psi}_{i}^{(2)}}{\mathrm{d} \bar{y}^{2}}-\mu_{i} \bar{\phi}^{k} \bar{\psi}_{k}^{(2)}=\mu_{1} \phi^{i k} \psi_{j}^{(1)} \psi_{k}^{(1)},
\end{gathered}
$$

(for $i=1,2,3$ ) and

$$
\bar{\psi}_{1}{ }^{(0)}=\bar{\psi}, \bar{\psi}_{1}{ }^{(p+1)}=\frac{\partial \bar{\psi}_{2}{ }^{(p)}}{\partial \tilde{y}}=\frac{\partial \bar{\psi}_{3}{ }^{(p)}}{\partial \bar{y}}=0
$$

(for $p=0,1,2, \ldots$ ) where, in perfect analogy with (2.17), the quantities $\bar{\phi}, \bar{\phi}^{k}, \bar{\phi}^{j k}$ are the values of $\phi\left(\psi_{1}, \psi_{2}, \psi_{3}\right)$ and its derivatives evaluated, now, at $\psi_{i}=\bar{\psi}_{i}{ }^{(0)}(\bar{y})$. One will also note that the differential Eqs. (2.24) for higher order terms $\bar{\psi}_{i}^{(p)}, p=1,2, \ldots$, are all linear.
At this point it would hardly be instructive to write down explicitly all the corresponding equations for the expansions at the right-hand boundary, $y=1$. Suffice it to remark that these will involve a set of perturbation coefficients $\psi_{i}^{(p)}(\underline{y})$, say, where

$$
\underline{y}=(1-y) / \epsilon \text {. }
$$

The relevant equations are readily obtained then from $(2.22),(2.24)$, and (2.25), by merely replacing the overbars by underbars.

In both the right- and left-hand boundary-layer expansions we must now place certain conditions on the $\bar{\psi}_{i}{ }^{(p)}$ (or $\underline{\psi}_{i}{ }^{(p)}$ ) for $\bar{y}$ (or $\underline{y}$ ) $\rightarrow \infty$, conditions which are to be provided by:

Matching of the boundary-layer and core expansions. According to the asymptoticmatching principle[17] we now insist that the expansions (2.22) shall match exactly with the core expansions $(2.15)$ in the sense that, for either $y \rightarrow 0$ or $\bar{y} \rightarrow \infty$,

$$
\begin{aligned}
\bar{\psi}_{i}^{(0)}(\bar{y})+\epsilon \bar{\psi}_{i}^{(1)}(\bar{y})+\ldots & \rightarrow \Psi_{i}^{(0)}(y) \\
& +\epsilon \Psi_{i}^{(1)}(y)+\ldots
\end{aligned}
$$

to terms of any order, $0\left(\epsilon^{p}\right), p=0,1,2, \ldots$, in $\epsilon$.

Following a method similar to one used previously[10], we next express the Taylor-series expansions in $y$ for the core functions:

$$
\begin{aligned}
\Psi_{i}^{(p)}(y)=\Psi_{i}^{(p)}(0)+y \frac{\mathrm{d} \Psi_{i}^{(p)}}{\mathrm{d} y} & (0) \\
& +\frac{y^{2}}{2} \frac{\mathrm{d}^{2} \Psi_{i}^{(p)}}{\mathrm{d} y^{2}}(0)+\ldots
\end{aligned}
$$

in terms of the coordinate $\bar{y}$ of (2.23), which, upon matching like powers of $\epsilon$ in (2.27) yields conditions of the form

$$
\begin{gathered}
\bar{\Delta}_{i}^{(p)}(\bar{y}) \equiv \bar{\psi}_{i}^{(p)}(\bar{y})-\sum_{m=0}^{p} \frac{(\bar{y})^{m}}{m !} \frac{\mathrm{d}^{m} \Psi_{i}^{(p-m)}}{\mathrm{d} y^{m}}(0) \\
\rightarrow 0, \text { for } \bar{y} \rightarrow \infty
\end{gathered}
$$

for $p=0,1,2, \ldots, i=1,2,3$. The quantities $\bar{\Delta}_{i}^{(p)}$ 
are essentially the differences or "mis-match" between terms of $0\left(\epsilon^{p}\right)$ in the core and the boundary-layer expansions, expressed as functions of $\bar{y}$. The corresponding differences for the righthand boundary are easily found to be

$$
\underline{\Delta}_{i}^{(p)}(\underline{y})=\underline{\psi}_{i}^{(p)}(\underline{y})-\sum_{m=0}^{p} \frac{(-\underline{y})^{m}}{m !} \frac{\mathrm{d}^{m} \Psi_{i}^{(p-m)}}{\mathrm{d} y^{m}}(1) .
$$

The matching requirements, $\bar{\Delta}$ (or $\underline{\Delta}$ ) $\rightarrow 0$ for $\bar{y}($ or $\underline{y}) \rightarrow \infty$, will provide us with certain necessary side conditions for the differential Eqs. (2.24) (or for their equivalent at the right boundary), as well as for the algebraic relations (2.18-2.20). In addition, however, we must also take account of the restrictions imposed by the integral relation of $(2.8)$. For this purpose, we note that an expansion of the form:

$$
\begin{aligned}
\Psi_{i}^{(0)}(y)+\bar{\Delta}_{i}^{(0)}(\bar{y})+\Delta_{i}^{(0)}(\underline{y})+\epsilon\left[\Psi_{i}^{(1)}(y)\right. \\
\left.+\bar{\Delta}_{i}^{(1)}(\bar{y})+\underline{\Delta}_{i}^{(1)}(\underline{y})\right]+\ldots
\end{aligned}
$$

where the $\Psi_{i}^{(p)}$ are the core functions of (2.14) and the $\Delta$ 's are defined by (2.28)-(2.29), should provide uniformily valid approximations for the functions $\psi_{i}$ on the entire interval $0 \leqslant y \leqslant 1$ to terms of any algebraic order in $\epsilon$. This follows from the fact, to be established below, that the functions $\Delta_{i}^{(p)}$ will be of an exponentially small order in $\epsilon$, for either $y=0(1)$ or $1-y=0(1)$, as $\epsilon \rightarrow 0$. Hence, it is permissible to use expansions of the above type for $\psi_{2}$ and $\psi_{3}$ in the integrand of (2.8) and, thus, apart from terms of a negligibly small order in $\epsilon$, we obtain a set of integral stoichiometric constraints

$$
\int_{0}^{1}\left[\Psi_{2}^{(0)}(y)+\Psi_{3}{ }^{(0)}(y)\right] \mathrm{d} y=1,
$$

and

$$
\int_{0}^{1}\left[\Psi_{2}^{(p)}(y)+\Psi_{3}^{(p)}(y)\right] \mathrm{d} y+\Delta^{(p-1)}=0,
$$

for $p=1,2, \ldots$, where

$$
\begin{aligned}
\Delta^{(p)}=\int_{0}^{\infty}\left[\bar{\Delta}_{2}^{(p)}(s)+\underline{\Delta}_{2}^{(p)}(s)\right. & +\bar{\Delta}_{3}^{(p)}(s) \\
& \left.+\underline{\Delta}_{3}^{(p)}(s)\right] \mathrm{d} s
\end{aligned}
$$

the $\bar{\Delta}_{i}^{(p)}$ and $\underline{\Delta}_{i}^{(p)}$ being the functions defined by (2.28) and (2.29). The interpretation of the relations (2.30) is evident; the first indicates merely that the equilibrium-core solutions must satisfy the stoichiometric restriction on the amount of non-transferable species present, while the succeeding relations for higher-order terms represent material balances between the core and the boundary-layer regions. Hence the $\Delta$ 's of (2.31) will be recognized as a type of "displacement thickness" common to other such boundary-layer analyses. With the conditions of (2.30), the formulation of the expansion problem is now essentially complete.

\subsection{Determination of the equilibrium core solutions and correction terms}

At this juncture we are able to propose a systematic technique for generating successively higher order terms in the boundary-layer and core expansions. As will be shown, the solution for these terms can be carried out implicitly in a rather general fashion and independently of the specific form of the kinetic function $\phi\left(\psi_{1}, \psi_{2}, \psi_{3}\right)$ in (2.9).

Beginning with the zeroth-order terms, we summarize the governing equations for the core function in the problem at hand, which are obtained from (2.18), (2.21) and (2.30):

$$
\begin{gathered}
\phi\left(\Psi_{1}{ }^{(0)}, \Psi_{2}{ }^{(0)}, \Psi_{3}{ }^{(0)}\right)=0, \\
\mu_{1} \Psi_{2}{ }^{(0)}-\mu_{2} \Psi_{1}{ }^{(0)}=\alpha_{12}^{(0)} y+\beta_{12}^{(0)}, \\
\mu_{2} \Psi_{3}{ }^{(0)}-\mu_{3} \Psi_{2}{ }^{(0)}=\beta_{23}^{(0)}\left(\alpha_{23}^{(0)}=0\right) \\
\int_{0}^{1}\left(\Psi_{2}{ }^{(0)}+\Psi_{3}{ }^{(0)}\right) \mathrm{d} y=1 .
\end{gathered}
$$

The first equation, which of course represents the equilibrium condition, together with the second and third equations are strictly algebraic relations in the unknown functions $\Psi_{i}^{\left({ }^{(0)}\right.}(y)$ and the constants $\alpha_{12}^{(0)}, \beta_{12}^{(0)}, \beta_{23}^{(0)}$. When applied at the boundaries $y=0,1$, these relations represent six equations in the nine unknown quantities $\Psi_{i}{ }^{\left({ }^{0}\right)}(0)$, $\Psi_{i}^{(0)}(1)(i=1,2,3) \alpha_{12}^{(0)}, \beta_{12}^{(0)}, \beta_{23}^{(0)}$. One further equation is provided by the integral of (2.32), and two more relations must be obtained from matching 
conditions like (2.28):

$$
\bar{\psi}_{i}^{(0)}(\bar{y}) \rightarrow \Psi_{i}^{(0)}(0), \text { for } \bar{y} \rightarrow \infty,
$$

for $i=1,2,3$, where the $\bar{\psi}_{i}^{(0)}(\bar{y})$ are the zerothorder terms in the (left-hand) boundary-layer expansions (2.22), which we recall must be chosen to satisfy the very first equations in (2.24) and (2.25). This latter requirement, together with the matching condition of (2.33), can be satisfied quite simply by taking the zeroth-order boundary-layer functions to be the following constants:

$$
\begin{gathered}
\bar{\psi}_{1}{ }^{(0)}(\bar{y}) \equiv \bar{\psi}_{i}^{(0)}(0)=\bar{\psi}=\Psi_{1}^{(0)}(0) \\
\bar{\psi}_{i}^{(0)}(\bar{y}) \equiv \Psi_{i}^{(0)}(0), i=2,3
\end{gathered}
$$

with similar equations at the left-hand boundary, $y=1(y=0)$. One may readily verify that, in addition to the boundary conditions in (2.25), the constants in (2.34) will identically satisfy the first set of differential equations in (2.24), since the term $\bar{\phi}$ appearing there becomes simply

$\bar{\phi} \equiv \phi\left(\Psi_{1}{ }^{(0)}(0), \Psi_{2}^{(0)}(0), \Psi_{3}{ }^{(0)}(0)\right) \equiv(\Phi)_{y=0}=0$

with the last equality resulting from the equilibrium condition of (2.32). Moreover, it is evident that the first equation of (2.34) and its equivalent at $y=1$ provide us with the two boundary conditions desired, on the core concentration field, $\Psi_{1}{ }^{(0)}(y)$, at $y=0$ and $y=1$.

To summarize, then, we have established the following result: The zero-order or equilibrium concentration fields in the core $\left(\Psi_{i}^{(0)}\right)$ are to satisfy the equilibrium relation, the kinetic and stoichiometric constraints (2.32), together with the exact boundary conditions (2.7) on the concentration field $\left(\psi_{1}\right)$ of the transferred species (A). On the other hand, we state most emphatically that in general one must not impose the zero-flux conditions (2.7) on the equilibrium core concentrations of the non-transferable species (B and C), because in the limit $\epsilon \rightarrow 0$, it will be shown that there is a net flux of these species to the core from the reaction boundary- layers, where, owing to local departure from chemical equilibrium, the reaction rates are $0(1 / \epsilon)$ over a region of thickness $0(\epsilon)$.

In line with the above remarks, Fig. 2 provides a qualitative sketch of the anticipated boundarylayer structure, as determined by the higherorder terms in (2.22). Although one could
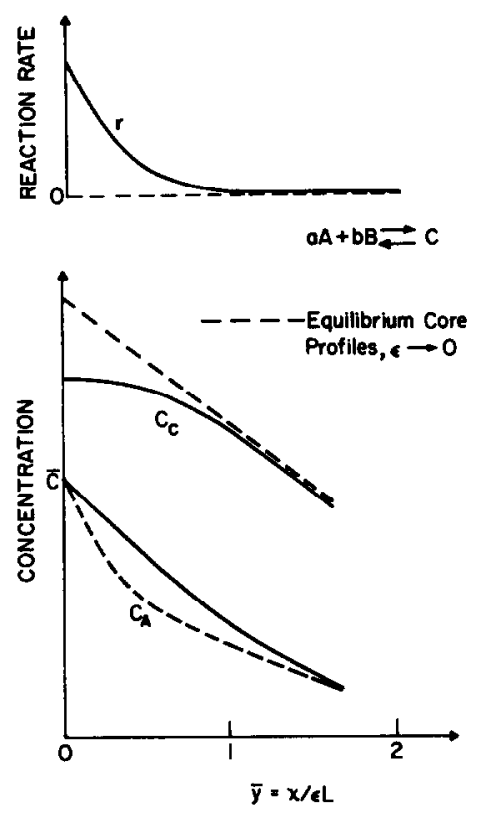

Fig. 2. Qualitative sketch of (left-hand) boundary-layer profiles for near-equilibrium reaction.

deduce asymptotic fluxes for $\epsilon \rightarrow 0$ from the equilibrium-core solutions (which as in past treatments $[13,20,21]$ could be done at this stage) the zeroth-order terms in (2.22) do not of course permit one to calculate mass transfer rates at the boundaries. Hence, we proceed with the derivation of some higher-order terms in the boundary-layer expansions.

As pointed out above, the differential equations of (2.24) for the higher-order terms in the boundary-layer expansion (2.22) are linear in the unknown function $\bar{\psi}_{i}^{(p)}(\bar{y})$ at any stage $(p=$ $1,2,3, \ldots)$ of the approximation. Also, a further simplification arises from the fact that the coeffi- 
cients in these differential equations, namely

$$
\bar{\phi}_{i} \equiv \frac{\partial \phi}{\partial \psi_{i}}\left(\bar{\psi}_{1}{ }^{(0)}, \bar{\psi}_{2}{ }^{(0)}, \bar{\psi}_{3}{ }^{(0)}\right)
$$

are all constants, simply because the zerothorder terms in their arguments are all constants, as given by (2.34); these constants are known in principle, once the equilibrium core solutions $\Psi_{i}^{(0)}$ are determined. Since, moreover, the side conditions of (2.25) and (2.28) are also linear, it is a relatively easy matter to derive a formal solution to the differential equations for the coefficients $\bar{\psi}_{i}^{(p)}(\bar{y})$ in (2.22).

The details of the derivation are deferred to a brief treatment in the Appendix. Here, we merely cite the final results obtained from the general solution, for the first- and second-order coefficients in the boundary-layer expansion (2.22).

First-order terms. The functions $\bar{\psi}_{i}^{(1)}$ in (2.22) are given by

$\bar{\psi}_{i}{ }^{(1)}(\bar{y})=-\frac{\mu_{i}}{\mu_{1}} \Psi_{1}^{(1)}(0) \mathrm{e}^{-\bar{\lambda} \bar{y}}+\bar{y} \frac{\mathrm{d} \Psi_{i}^{(0)}}{\mathrm{d} y}(0)+\Psi_{i}^{(1)}(0)$

for $i=1,2,3$, where

$$
\begin{gathered}
\bar{\lambda}^{2}=\bar{\phi}^{j} \mu_{j}(>0), \\
\Psi_{1}^{(1)}(0)=-\frac{\mu_{1}}{\bar{\lambda} \mu_{2}} \frac{\mathrm{d} \Psi_{2}{ }^{(0)}}{\mathrm{d} y}(0) \equiv-\frac{\mu_{1}}{\bar{\lambda} \mu_{3}} \frac{\mathrm{d} \Psi_{3}^{(0)}}{\mathrm{d} y}(0),
\end{gathered}
$$

and the $\Psi_{i}^{(p)}, p=0,1$, refer of course to core expansion coefficients. One can readily verify that (2.35) satisfies the appropriate differential equations and boundary conditions of (2.24) and (2.25), provided the boundary value of the firstorder term in the core expansion for the transferred species (A) is chosen in accordance with (2.37). (This also follows from the general analysis in the Appendix.) Similar expressions hold for the right-hand boundary at $y=1$, whence

$$
\Psi_{1}^{(1)}(1)=\frac{\mu_{1}}{\underline{\lambda} \mu_{2}} \frac{\mathrm{d} \Psi_{2}^{(0)}}{\mathrm{d} y}(1) \equiv \frac{\mu_{1}}{\underline{\lambda} \mu_{3}} \frac{\mathrm{d} \Psi_{3}^{(0)}}{\mathrm{d} y}(1),(2.38)
$$

where

$$
\underline{\lambda}^{2}=\underline{\phi}^{j} \mu_{j}(>0)
$$

which are of course equivalent to (2.36) and (2.37). Finally, the first-order displacement thickness $\Delta^{(1)}$ of (2.31) is given by

$$
\begin{aligned}
\frac{\Delta^{(1)}}{\mu_{2}+\mu_{3}} & =\frac{1}{\mu_{2}}\left[\frac{1}{\bar{\lambda}^{2}} \frac{\mathrm{d} \Psi_{2}^{(0)}}{\mathrm{d} y}(0)-\frac{1}{\underline{\lambda}^{2}} \frac{\mathrm{d} \Psi_{2}^{(0)}}{\mathrm{d} y}(1)\right] \\
& =\frac{1}{\mu_{3}}\left[\frac{1}{\bar{\lambda}^{2}} \frac{\mathrm{d} \Psi_{3}^{(0)}}{\mathrm{d} y}(0)-\frac{1}{\underline{\Lambda}^{2}} \frac{\mathrm{d} \Psi_{3}^{(0)}}{\mathrm{d} y}(1)\right] .
\end{aligned}
$$

The reader will note that we have required both the quantities $\lambda^{2}$ defined by (2.36) and (2.39) to be non-negative. By imposing this restriction, which is essential to the mathematical analysis, we have in effect narrowed the heretofore somewhat abstract class of "kinetic functions" $\phi$ to which the analysis can be considered directly applicable. In particular, one will recognize that this restriction follows from the so-called principle of "microscopic reversibility," of the thermodynamics of irreversible processes, a principle which has been invoked frequently in other such problems involving chemical kinetics with diffusion [6].

With the above results in hand, one now can in principle calculate the first-order correction terms $\Psi_{i}^{(1)}$ in the core expansion (2.14). Next, we cite the corresponding results for the

Second-order terms. Here we find

$$
\begin{gathered}
\bar{\psi}_{i}^{(2)}(\bar{y})=\frac{\mu_{i} \Psi_{1}^{(1)}(0)}{\mu_{1} \bar{\lambda}} \bar{\phi}^{j k} \mu_{j}\left(\left[\frac{\bar{y}^{2}}{2} \frac{\mathrm{d} \Psi_{k}{ }^{(0)}}{\mathrm{d} y}(0)\right.\right. \\
\left.+\bar{y}\left\{\Psi_{k}^{(1)}(0)+\frac{1}{2 \bar{\lambda}} \frac{\mathrm{d} \Psi_{k}^{(0)}}{\mathrm{d} y}(0)\right\}-\frac{\mu_{k}}{3 \mu_{1} \bar{\lambda}} \Psi_{1}^{(1)}(0)\right] \\
\left.\mathrm{e}^{-\bar{\lambda} \bar{y}}+\frac{\mu_{k}}{3 \mu_{1} \lambda} \Psi_{1}^{(1)}(0) \mathrm{e}^{-2 \bar{\lambda} \bar{y}}\right)-\frac{\mu_{i}}{\mu_{1}} \Psi_{1}{ }^{(2)}(0) \mathrm{e}^{-\bar{\lambda} \bar{y}} \\
+\Psi_{i}^{(2)}(0)+\bar{y} \frac{\mathrm{d} \Psi_{i}^{(1)}}{\mathrm{d} y}(0)+\frac{\bar{y}^{2}}{2} \frac{\mathrm{d}^{2} \Psi_{i}^{(0)}}{\mathrm{d} y^{2}}(0),
\end{gathered}
$$


for the left-hand boundary, where

$$
\begin{aligned}
& \Psi_{1}^{(2)}(0)=-\frac{\mu_{1}}{\bar{\lambda} \mu_{2}} \frac{\mathrm{d} \Psi_{2}^{(1)}}{\mathrm{d} y}(0)-\frac{\Psi_{1}^{(1)}(0)}{\bar{\lambda}^{2}} \phi^{j k} \mu_{j} \\
& \left\{\frac{1}{2 \bar{\lambda}} \frac{\mathrm{d} \Psi_{k}^{(0)}}{\mathrm{d} y}(0)+\Psi_{k}^{(1)}(0)-\frac{\mu_{k} \Psi_{1}^{(1)}}{3 \mu_{1}}(0)\right\},
\end{aligned}
$$

and, from similar results at the right-hand boundary,

$\Psi_{1}^{(2)}(1)=\frac{\mu_{1}}{\underline{\lambda} \mu_{2}} \frac{\mathrm{d} \Psi_{2}{ }^{(1)}}{\mathrm{d} y}(1)+\Psi_{1}^{(1)}(1) \frac{\phi^{3 k} \mu_{j}}{\underline{\Lambda}^{2}}$

$\left\{\frac{1}{2 \underline{\underline{\lambda}}} \frac{\mathrm{d} \Psi_{k}^{(0)}}{\mathrm{d} y}(1)-\Psi_{k}^{(1)}(1)+\frac{\mu_{k}}{3 \mu_{1}} \Psi_{1}^{(1)}(1)\right\}$.

Again, one could use the boundary values at $y=0,1$ prescribed by (2.41) and (2.42) to effect the calculation of second-order terms $\Psi_{i}^{\left({ }^{2)}\right.}$ in the core expansion, and so forth for higher-order terms.

From the analysis given in the Appendix it follows that one can obtain flux rates to terms $O\left(\epsilon^{\nu}\right)$ from

$$
\begin{gathered}
\frac{L N_{A}}{a C_{0} D_{A}} \equiv-\frac{\mathrm{d} \psi \psi_{1}}{\mathrm{~d} y}(0)=-\frac{1}{\epsilon}\left[\frac{\mathrm{d}}{\mathrm{d} y} \sum_{m=0}^{p} \bar{\psi}_{1}^{(m)}(\bar{y}) \epsilon^{m}\right. \\
\left.+0\left(\epsilon^{p+1}\right)\right]_{\bar{y}=0} \\
=-\sum_{m=0}^{p}\left[\frac{\mathrm{d} \Psi_{1}^{(m)}}{\mathrm{d} y}(0)-\frac{\mu_{1}}{\mu_{2}} \frac{\mathrm{d} \Psi_{2}^{(m)}}{\mathrm{d} y}(0)\right] \epsilon^{m}+0\left(\epsilon^{(p+1)}\right) \\
\equiv \sum_{m=0}^{p} \frac{\alpha_{12}^{(m)}}{\mu_{2}} \epsilon^{m}+0\left(\epsilon^{p+1}\right)
\end{gathered}
$$

To provide some indication of the general utility and the limitations of this type of analysis, the present results will now be applied to some rather simple special cases.

\section{APPLICATION AND DISCUSSION OF THE RESULTS}

At this point, we shall treat two mathematical examples where the exact analytic solutions can also be obtained, and a third example for which some numerical solutions are available for comparison. Another application to an interesting physical system, is anticipated for a later publication [2]. The following examples will provide some basis for assessing the range of applicability and the limitations on the expansions.

\subsection{Linear or pseudo-first-order kinetics}

Here we suppose that we have a single chemical reaction among an arbitrary number of diffusing species, for which the kinetic function corresponding to (2.6) has the (dimensionless) form

$$
\phi=\phi^{j} \psi_{j}
$$

where the $\phi^{j}$ are constants and the sum implied in (3.1) is taken over all species, $j=1,2,3, \ldots$ Considering the case where only one species, corresponding, say, to $j=1$, is transferred, we recover a problem which is mathematically similar to that considered previously by Friedlander and Keller [8]. As pointed out by these authors, the assumption of linear kinetics is in general valid in the limit of small departures from equilibrium, where the driving force for mass transfer $\bar{\psi}-\psi \rightarrow 0$. In this case, the governing differential equations have generally a linear form, identical with that of the second equation in (2.24), and the boundary conditions are the same here as those in (2.7).

By methods similar to those employed in the Appendix, one can readily derive the exact solution to the linear problem; omitting the details here, we merely cite the principal result, which gives the quantity $S$ of (2.14) as

$$
S=\frac{1}{1-\left(\mu_{1} \phi^{1} / \lambda^{2}\right) F}
$$

where

$$
F=F(\lambda / \epsilon)=1-\frac{2 \epsilon}{\lambda}\left[\frac{\sinh (\lambda / \epsilon)}{\cosh (\lambda / \epsilon)+1}\right]
$$

and

$$
\lambda^{2} \equiv(\lambda)^{2}=\mu_{j} \phi^{j}
$$

Although the present analysis is slightly more general, the function $F(\lambda / \epsilon)$ in (3.3) is identical with the function introduced in the previous 
analysis of Friedlander and Keller [8], as a "correction factor for departure from chemical equilibrium" (which is displayed graphically as Fig. 2 of their paper).

For $\epsilon \rightarrow 0$, one has asymptotically that

$$
F \sim 1-2 \epsilon / \lambda
$$

and hence, by (3.2), that

$$
S \sim\left(\lambda / \lambda_{1}\right)^{2}\left\{1+2\left[\left(\frac{\lambda}{\lambda_{1}}\right)^{2}-1\right] \frac{\epsilon}{\lambda}\right\}^{1},
$$

to terms $0\left(\mathrm{e}^{-\lambda / \epsilon}\right)$, where

$$
\lambda_{1}{ }^{2}=\lambda^{2}-\mu_{1} \phi^{1} .
$$

Now, it is a relatively easy matter to show that the asymptotic series (2.43), generated by the analysis of Section 2 , is equivalent to the "binomial" expansion of (3.5) for small $\epsilon$, an expansion with a finite radius of convergence $\epsilon_{m}$, say, which is given by

$$
\epsilon_{m}=\frac{\lambda}{2\left[\left(\lambda / \lambda_{1}\right)^{2}-1\right]} .
$$

Thus, for the special case at hand we can state the range of validity of the asymptotic expansion (2.43) as $\epsilon<\epsilon_{m}$, and, moreover, we can easily assess its accuracy within this range. For example, one concludes that the first three terms of (2.43) will provide an approximation which differs from the exact result of (3.2) by less than one percent whenever $\epsilon \leqslant 0.2 \mathrm{~min}$ $\left(\lambda, \epsilon_{m}\right)$. In the more general case of a non-linear kinetic function we should not expect to obtain any such simple criteria and, to illustrate this we turn to the second example.

\subsection{Second-order kinetics with two species}

Let us now apply the results of Section 2 to an elementary reaction between the transferred and carrier species:

$$
2 \mathrm{~A} \rightleftarrows 2 \mathrm{C}
$$

in which both the forward and reverse reactions are assumed to be second-order. To employ the formalism of Section 2 we merely eliminate the species B in (2.1) and let the subscript " 2 " refer now to the carrier species C. Also, since there are now no redundant flux conditions in (2.4), the integral constraint (2.5) and its successors are no longer in effect. Therefore, the kinetic function can be written in the (dimensionless) form

$$
\phi=\left(\psi_{1}\right)^{2}-(1 / K)\left(\psi_{2}\right)^{2}
$$

(where $K(>0)$ is an "equilibrium constant"). Thus, the partial derivatives, of the type occurring in (2.17), are given for arbitrary $\psi_{1}$ by

$$
\begin{gathered}
\phi^{1}=2 \psi_{1}, \phi^{2}=-\frac{2}{K} \psi_{2}, \\
\phi^{11}=1, \phi^{22}=-1 / K, \phi^{12} \equiv \phi^{21}=0,
\end{gathered}
$$

with all higher derivatives vanishing, of course. One easily deduces then that the core Eqs., (2.18)-(2.20), become simply

$$
\Psi_{2}^{(p)}(y)=\Psi_{1}^{(p)}(y) \vee K,
$$

for $p=0,1,2, \ldots$, and accordingly that the $\Psi_{i}^{(p)}(y)$ are the linear functions:

$$
\frac{1}{\sqrt{ } K} \Psi_{2}^{(p)}(y)=\Psi_{1}^{(p)}(y)=\frac{\alpha_{21}^{(p)} y+\beta_{21}^{(p)}}{\mu_{2}-\mu_{1} \sqrt{ } K},
$$

as given by (2.21). Hence, the constants $\alpha, \beta$ are simply related to the boundary values of $\Psi_{1}^{(p)}(y)$ by

$$
\beta_{21}^{(p)}=\left(\mu_{2}-\mu_{1} \vee K\right) \Psi_{1}^{(p)}(0),
$$

and

$$
\alpha_{21}^{(p)}=\left(\mu_{2}-\mu_{1} \vee K\right)\left[\Psi_{1}^{(p)}(1)-\Psi_{1}^{(p)}(0)\right],
$$

while the constants $\lambda^{2}$ of (2.36) and (2.39) are given by

$$
\bar{\lambda}^{2} / \bar{\psi}=\underline{\lambda}^{2} / \underline{\psi}=2\left(\mu_{1} \sqrt{ } K-\mu_{2}\right)
$$

(which, we recall, are non-negative quantities). 
It is a straight-forward procedure to apply the boundary conditions $(2.34),(2.37),(2.38),(2.41)$ and (2.42), and to thereby effect a sequential solution of (3.9) and (3.10), which, with (2.43), finally yields for the quantity $S$ of (2.14)

$S=(1+\rho)\left\{1+S^{(1)} \epsilon+S^{(2)} \epsilon^{2}+0\left(\epsilon^{3}\right)\right\}$,

where

$$
\begin{array}{r}
S^{(1)}=-\rho\left(\frac{1}{\bar{\lambda}}+\frac{1}{\underline{\lambda}}\right), \\
S^{(2)}=\left[S^{(1)}\right]^{2}-\frac{\mu_{1}}{6}\left(\frac{1}{\bar{\lambda}^{4}}-\frac{1}{\underline{\lambda}^{4}}\right)(\bar{\psi}-\underline{\psi})
\end{array}
$$

with

$$
(1+\rho)(5+4 \rho) \text {, }
$$

$$
\rho=-\frac{\mu_{1} \vee K}{\mu_{2}}
$$

For the special case $\rho=1$ in (3.13) it is also possible to derive an exact solution for the problem at hand, since the appropriate integral relation of (2.11) can then be expressed as

$$
\mu_{2} \psi_{1}-\mu_{1} \psi_{2} \equiv \mu_{2}\left(\psi_{1}+\frac{1}{\sqrt{ } K} \psi_{2}\right)=\alpha_{21} y+\beta_{21}
$$

hence, the kinetic function of (3.6) reduces to

$$
\phi=\left(\alpha_{21} y+\beta_{21}\right)\left(\psi_{1}-\frac{\psi_{2}}{\sqrt{ } K}\right) / \mu_{2},
$$

and, by (2.6), one obtains a linear differential equation

$$
\epsilon^{2} \frac{\mathrm{d}^{2} \psi}{\mathrm{d} y^{2}}-\frac{2 \mu_{1}}{\mu_{2}}\left(\beta_{21}+\alpha_{21} y\right) \psi=0,
$$

for the function

$$
\psi \equiv \psi_{1}-\frac{1}{\sqrt{ } K} \psi_{2}
$$

The general solution to this differential equation, which is related to the so-called Airy equation, can be expressed in terms of modified Bessel functions [11] of the type $I_{ \pm 1 / 3}$. With the appro- priate boundary conditions from (2.7), this leads to a pair of implicit, transcendental equations for the constants $\alpha_{21}$ and $\beta_{21}$. Without writing down these rather complex relations here, we merely note that for $\epsilon \rightarrow 0$ they can be solved asymptotically, by means of well-known, "semiconvergent" asymptotic expansions [19] (for large arguments) of the relevant Bessel function, $I_{\nu}, K_{\nu}$, to yield asymptotic expansion for $\alpha_{21}$ and $\beta_{21}$. The resulting expansion for $S$ is found to agree, to terms $0\left(\epsilon^{2}\right)$, with our expansions of (3.13), when $\rho=1$ there.

Although the above comparison provides a partial confirmation of our expansion (and also shows that it is a semi-convergent series for the present example), we must exclude the exceptional case when either of the boundary values $\bar{\psi}$ or $\psi$ of (2.7) vanishes. For then the asymptotic expansion, as derived from the exact solution, changes its character radically, and for $\bar{\psi}>\underline{\psi}=0$ we obtain instead of (3.12)

$$
\begin{aligned}
S & =2\left\{1-\frac{0.866 . . \epsilon^{2 / 3}}{\left(4 \bar{\psi} \mu_{1}\right)^{1 / 3}}-\frac{\epsilon}{2 \sqrt{ }\left(\bar{\psi} \mu_{1}\right)}\right. \\
& \left.+\frac{2}{3}\left[\frac{0.866 \ldots}{\left(4 \bar{\psi} \mu_{1}\right)^{1 / 3}}\right]^{2} \epsilon^{4 / 3}+0\left(\epsilon^{5 / 3}\right)\right\},
\end{aligned}
$$

where, incidentially, the number $0.866 \ldots$ is the root $z$ of the equation

$$
z A i^{\prime}(z)+A i(z)=0,
$$

with $A i$ and $A i^{\prime}$ denoting, respectively, the Airy function and its derivatives [11].

\subsection{Second-order boundary layers}

In the special case considered above, one sees immediately that the expansion of (3.12) becomes singular whenever $\lambda$ (i.e. $\lambda$ or $\bar{\lambda}$ ) vanishes. Furthermore, the entire analysis of Section 2 , where we merely stipulated $\lambda^{2}>0$, will always fail in the limiting case $\lambda=0$. This is due to the fact that, among the set of admissible "equilibrium" concentrations, we can have a subset where one or more of the concentrations are zero and where, consequently, the kinetic 
function $\phi$ as well as its first derivatives $\phi^{j}$ vanish.

We may expect to encounter this difficulty for certain reactions whenever one boundary concentration, say, $\bar{\psi}$, at the right-hand boundary, is fixed and we achieve the maximum "driving force", in the limit $\psi \rightarrow 0$ at the other boundary. In this limit, we would simultaneously achieve a state of chemical equilibrium at the right-hand boundary, irrespective of the magnitude of our perturbation parameter $\epsilon$. As a consequence there would obtain, in the limit $\epsilon \rightarrow 0$, a "weak" reaction zone at the right-hand boundary with a type of "second-order" boundary layer, such that the analysis of Section 2 is no longer applicable there and must be modified. Thus, if we suppose that for $\psi=0$, the second derivatives $\phi^{i j}$ are not all zero, then we must conclude that the right-hand boundary layer will be of a thickness $0\left(\epsilon^{2 / 3}\right)$ for $\epsilon \rightarrow 0$. Therefore, instead of the expansions postulated in Section 2, one has to consider now a core expansion of the form

$$
\psi=\Psi^{(0)}(y)+\epsilon^{2 / 3} \Psi^{(2)}(y)+\epsilon \Psi^{(3)}(y)+0\left(\epsilon^{4 / 3}\right)
$$

which for $\Psi_{1}{ }^{(0)}(1)=\psi=0$, will match with a right-hand boundary-layer expansion of the form.

$$
\psi=\epsilon^{2 / 3} \underline{\psi}^{(2)}(\underline{y})+\epsilon \underline{\psi}^{(3)}(\underline{y})+0\left(\epsilon^{4 / 3}\right)
$$

where

$$
\underline{y} \equiv \frac{1-y}{\epsilon^{2 / 3}} .
$$

In this way, a perturbation analysis similar to that of Section 2, leads now to the non-linear differential equation

$$
\frac{\mathrm{d}^{2} \psi_{i}^{(2)}}{\mathrm{d}^{2}}-\mu_{i} \underline{\phi}^{j k} \psi_{j}^{(2)} \underline{\psi}_{k}^{(2)}=0,
$$

for the lowest-order term in (3.16). Although the left-hand boundary-layer expansion is still the same as in (2.2), the difficulties inherent in solving (3.17), and subsequent higher-order equations, would appear to preclude the kind of analytic treatment we have been able to give in Section 2.

\subsection{Equal diffusivities of the non-transferable species}

Whenever the diffusivities of the species $\mathbf{B}$ and $\mathbf{C}$ in (2.1) are equal, i.e. when

$$
D_{C}=D_{B^{\prime}}
$$

some important simplifications arise in the analysis of Section 2. In particular, we may take

$$
D^{*}=D_{A}, \mu_{1} \equiv 1, \mu_{2}=-\mu_{3}=D_{A} / D_{B}=D_{A} / D_{C},
$$

in (2.9), and, by virtue of (2.13) and the kinetic constraint (2.11) on B and C, the stoichometric constraint of (2.8) yields

$$
\beta_{23} / \mu_{2}=\psi_{2}+\psi_{3} \equiv 1, \text { for } 0 \leqslant y \leqslant 1 .
$$

By the same reasoning, applied to core expansions of the type (2.14) and (2.15), one finds that

$$
\beta_{23}^{(p)} / \mu_{2}=\Psi_{2}^{(p)}+\Psi_{3}^{(p)} \equiv\left\{\begin{array}{l}
1 \text { for } p=0 \\
0 \text { for } p=1,2,3, \ldots
\end{array}\right.
$$

for $0<y<1$, and, therefore, that the "displacement thickness" $\Delta^{(p)}$ of (2.31) vanishes for $p=1,2, \ldots$ That is, the equilibrium terms $\Psi_{2}{ }^{(0)}$, $\Psi_{3}{ }^{(0)}$, satisfy exactly the original kinetic and stoichiometric constraints, and the succeeding terms $\Psi_{2}{ }^{(p)}, \Psi_{3}{ }^{(p)}, p=1,2, \ldots$, make no further contributions in the equations of constraint. This introduces a considerable simplification into the analysis, since the integral constraints of (2.30) are now satisfied automatically at any stage of the perturbation, $p=1,2, \ldots$

Moreover, the relations (3.21) lead to several simplifications in the equations for the core functions, which permits us to derive some rather explicit formulae for the determination of 
both the zeroth- and first-order terms for mass flux. In particular, the Eqs. (2.32) for the equilibrium terms reduce to

$$
\phi\left(\Psi_{1}{ }^{(0)}, \Psi_{2}{ }^{(0)}, 1-\Psi_{2}{ }^{(0)}\right)=0
$$

with

$$
\Psi_{2}^{(0)}=\mu_{2} \Psi_{1}^{(0)}+\alpha_{12}^{(0)} y+\beta_{12}^{(0)}
$$

which, when applied at the boundaries $y=0,1$, logether with the respective boundary values $\bar{\psi}, \underline{\psi}$ for $\Psi_{1}{ }^{(0)}$, provide two equations for $\beta_{12}^{(0)}$ and, the quantity of primary interest, $\alpha_{12}^{(0)}$.

Furthermore, the Eqs. (2.19) and (2.21) for the first-order terms reduce now to

$$
\begin{gathered}
\Psi_{2}^{(1)}=-\Psi_{3}^{(1)} \\
\Phi^{j} \Psi_{j}^{(1)}=\Phi^{1} \Psi_{1}{ }^{(1)}+\left(\Phi^{2}-\Phi^{3}\right) \Psi_{2}{ }^{(1)}=0 \\
\Psi_{2}^{(1)}=\mu_{2} \Psi_{1}{ }^{(1)}+\alpha_{12}^{(1)} y+\beta_{12}^{(1)}
\end{gathered}
$$

which can easily be solved for $\Psi_{1}^{(1)}$, to yield

$$
\Psi_{1}^{(1)}(y)=\frac{\Phi^{1}-\Lambda^{2}}{\mu_{2} \Lambda^{2}}\left(\alpha_{12}^{(1)} y+\beta_{12}^{(1)}\right)
$$

where the function $\Lambda$, defined by,

$$
\Lambda^{2} \equiv(\Lambda)^{2}=\Phi^{j} \mu_{j}=\Phi^{1}+\mu_{2}\left(\Phi^{2}-\Phi^{3}\right),
$$

takes on, at $y=0,1$, the respective values $\bar{\lambda}^{2}$, $\lambda^{2}$, given by (2.36) and (2.39).

We can now obtain a fairly explicit expression for the constants $\alpha_{12}^{(1)}$ in (3.24) by application of (3.24) at $y=0,1$. Thus, by first making use of the boundary values (2.37) and (2.38), and the equilibrium relations (3.22) (more precisely, of their $y$-derivatives), we find that

$$
\Psi_{1}^{(1)}(0)=-\bar{\phi}^{1} \alpha_{12}^{(0)} / \bar{\lambda}^{3} \mu_{2}
$$

and

$$
\Psi_{1}^{(1)}(1)=\underline{\phi}_{1} \alpha_{12}^{(0)} / \underline{\lambda}^{3} \mu_{2}
$$

where $\bar{\phi}^{1}, \phi^{1}$, the boundary values of $\Phi^{1}$, are of course the differential coefficients (for $i=1$ ) of the type introduced in Eqs. (2.24). Next, by evaluating the right-hand side of (3.24) at the boundaries and by equating it to (3.25), we find the remarkably simple relation

$\alpha_{12}^{(1)} / \alpha_{12}^{(0)}=-\left[\frac{\bar{\phi}^{1}}{\lambda\left(\bar{\lambda}^{2}-\bar{\phi}^{1}\right)}+\frac{\underline{\phi}_{1}}{\underline{\lambda}\left(\underline{\lambda}^{2}-\underline{\phi}^{1}\right)}\right]$.

Hence once the form of the kinetic function $\phi$ is specified, permitting one to solve the equilibrium relations of (3.22) at $y=0,1$ for $\alpha_{12}^{(0)}$ and also to determine the quantities $\bar{\lambda}, \bar{\phi}^{1}, \ldots$, we see by (2.43) and (3.26) that the first-order correction for mass flux immediately follows. In fact, if we let $S^{(0)}$ denote the value of the Sherwood number of (2.14) for $\epsilon=0$, we have that

$$
S / S^{(0)}=1+\left(\alpha_{12}^{(1)} / \alpha_{12}^{(0)}\right) \epsilon+0\left(\epsilon^{2}\right)
$$

where $\alpha_{12}^{(1)} / \alpha_{12}^{(0)}$ is of course given directly by (3.26).

\subsection{Comparison with a numerical solution}

As an illustrative example for the case of equal diffusivities considered in the preceding paragraphs, we apply the results to a model for the diffusion of oxygen through hemoglobin solutions. Experimental measurements on this system have been made by Scholander and Hemmingsen [12] and some numerical solutions of the relevant differential equations have recently been performed by Kutchai, Jacquez, and Mathers $\dagger$.

The reaction of interest can be represented by equation (2.1), with $a=b=1$, and with A representing oxygen, $\mathbf{B}$, hemoglobin and $\mathbf{C}$, oxyhemoglobin, the "carrier". The reaction kinetics, although complex, can be reasonably well represented by

$$
r=k_{1} C_{A} C_{B}-k_{2} C_{C},
$$

or, in the notation (2.6)-(2.9) of the present work, by

$$
\phi=\psi_{1} \psi_{2}-\psi_{3} / K_{0}
$$

†Unpublished manuscript of a paper. Non-Equilibrium Facilitated Oxygen Transport in Hemoglobin Solutions, to appear, which the present authors gratefully acknowledge. 
if one takes

$$
r^{*} \equiv k_{1} C_{0}^{2}, K_{0} \equiv k_{1} C_{0} / k_{2} .
$$

If we further adopt (3.19), then the analysis of the preceding section is directly applicable, and, in particular, it is a straight-forward matter to solve the equilibrium relation (3.22) for the special form (3.29) of the kinetic function. This leads to some elementary quadratic equations for the equilibrium-core solutions $\Psi_{i}{ }^{(0)}$, which we shall not trouble to reproduce here. Rather more interesting is the solution for $\alpha_{12}^{(0)}$, which is obtained by application of (3.22) at $y=0,1$, and which can be expressed as

$\alpha_{21}^{(0)} / \mu_{2}=(\bar{\psi}-\underline{\psi})\left[\frac{\sigma^{2}}{(1+\bar{K})(1+\underline{K})}+1\right]$,

where

$\bar{K}=K_{0} \bar{\psi}=k_{1} \bar{C}_{A} / k_{2}, \underline{K}=K_{0} \Psi=k_{1} \underline{C}_{A} / k_{2}$,

and

$$
\sigma^{2} \equiv(\sigma)^{2}=K_{0} / \mu_{2}=k_{1} C_{0} D_{B} / k_{2} D_{A}
$$

Thus, the equilibrium Sherwood number, corresponding to $\epsilon=0$, can be expressed compactly as

where

$$
S^{(0)} \equiv \frac{\alpha_{12}^{(0)}}{\mu_{2}(\bar{\psi}-\underline{\psi})}=\bar{Z} \underline{Z}+1
$$

$$
\bar{Z}=\sigma /(1+\bar{K}), \underline{Z}=\sigma /(1+\underline{K})
$$

To obtain the first-order term in $\epsilon$ we note that, for the case at hand, the derivatives in (2.17) are

$$
\Phi^{1}=\bar{\Psi}_{2}{ }^{(0)}(y), \Phi^{2}=\Psi_{1}^{(0)}(y), \Phi^{3}=-1 / K_{0},
$$

which, with the appropriate boundary values of $\Psi_{i}^{(0)}$, give

$\bar{\phi}^{1}=1 /(1+\bar{K}), \bar{\phi}^{2}=\bar{\psi}, \bar{\phi}^{3}=-1 / K_{0}$,

and, hence,

$$
\bar{\lambda}^{2} \equiv \bar{\phi}^{j} \mu_{j}=\left(1+\bar{Z}^{2}\right) / \sigma \bar{Z},
$$

together with a similar expression for $\underline{\lambda}^{2}$ in terms of $\underline{Z}$. Thus, we find readily by (3.26) and (3.27) that

$$
S / S^{(0)}=1-[G(\bar{Z})+G(\underline{Z})] \sigma^{1 / 2} \epsilon+0\left(\epsilon^{2}\right),
$$

where

$$
\begin{aligned}
G(Z) & =\vee\left(Z^{5} /\left(1+Z^{2}\right)\right), \\
\epsilon & =\vee\left(D_{A} / L^{2} k_{1} C_{0}\right),
\end{aligned}
$$

and $\bar{Z}, Z$, and $\sigma$ are defined by (3.31) and (3.32).

As mentioned above, Kutchai et al. have obtained numerical solutions for the differential equations of the problem at hand and the table shown here provides a comparison of some of their numerical results to those obtained from the first-order asymptotic expansion of (3.35). The comparison is based on their values of the "facilitation" $f$, which is defined as fractional increase in oxygen flux due to the presence of chemical reaction and which, therefore, can be expressed as

$$
f=S-1
$$

As indicated in the Table, the given values of $f$ correspond to three different membrane thicknesses $L$ and two levels of oxygen concentration $\underline{C}$ at one boundary, with all other parameters fixed. For smaller values of $L$ than those shown in the Table the first-order expansion of (3.35) becomes progressively more inaccurate, as one would expect from the expression for the parameter $\epsilon$ in (3.35).

Also, one can immediately see by $(3.35)$ that, as in the previous examples, the formal expansion parameter $\epsilon$ is not the sole criterion for departure from equilibrium. Rather, one concludes from the form of the first-order correction term in (3.35), that it cannot be small relative to unity if the equilibrium flux ratio $S^{(0)}$, as given by (3.32), is large. Indeed, this is borne out by a comparison with other numerical examples of Kutchai et al. where, for large $f$, the two-term expansion (3.35), is wholly inapplicable. In such cases, the first-order term in (3.35) serves mainly 
Table 1. Comparison of the first-order asymptotic expansion (3.35) to a numerical solution, for the facilitation of oxygen diffusion by hemoglobin. The parameters are: (A: oxygen, B: hemoglobin, C: oxyhemoglobin) Diffusivities (cm²/sec): $D_{A}=6 \times 10^{-6}, D_{B}=D_{C}=5 \times 10^{-8}$. Rate Constants in (3.28): $k_{1}=1.8 \times 10^{9} \mathrm{~cm}^{3} /$ mole sec, $k_{2}=67.3 \mathrm{sec}^{-1}$. Concentrations (moles $/ \mathrm{cm}^{3}$ ): $C_{0}=2 \times 10^{-5}, \bar{C}=1.7 \times 10^{-7}$, Case I: $\underline{C}=0$. Case II: $\underline{C}=6.8 \times 10^{-8}$

\begin{tabular}{ccccc}
\hline & \multicolumn{4}{c}{ Facilitation, $f$} \\
\cline { 2 - 6 } Membrane thickness & \multicolumn{2}{c}{ Case I } & \multicolumn{2}{c}{ Case II } \\
\cline { 2 - 6 }$L(\mu)$ & Eq. (3.35) & Exact & Eq. (3.35) & Exact \\
\hline 2 & 0.322 & 0.500 & 0.191 & 0.234 \\
5 & 0.610 & 0.646 & 0.247 & 0.263 \\
25 & 0.764 & 0.769 & 0.277 & 0.280 \\
\hline$\infty$ (equilibrium) & \multicolumn{3}{c}{0.803} & \multicolumn{2}{c}{0.285} \\
\hline
\end{tabular}

as a criterion for indicating the departure from reaction equilibrium. In general, one might expect a quantity such as $\epsilon \alpha_{12}^{(1)} / \alpha_{12}^{(0)}$, as given by (3.26), to provide a reasonable estimate of the departure from reaction equilibrium even though it was derived for the special case of equal diffusivities of the non-transferred species.

\subsection{Conclusions}

The asymptotic expansion of the present analysis (2.43) would in general permit one to compute membrane-diffusion rates, to terms of the second-order in the kinetic parameter $\epsilon$, for rapid reversible reactions, where $\epsilon \ll 1$. The limiting diffusion rate for $\epsilon \rightarrow 0$ is completely determined by the equilibrium-core concentrations, which satisfy the reaction equilibrium, the kinetic and stoichiometric constraints, (2.32), and the exact boundary conditions on the transferred species, (2.7). Higher-order corrections in $\epsilon$ could in principle be computed from the solutions for corresponding terms in the coreconcentration fields which are governed by (2.19)-(2.21), (2.30), and (2.37)-(2.42). It will be recalled that the solution to these equations would mainly involve algebraic operations and quadratures.

In closing, it might be noted that there are some perhaps obvious, but potentially quite useful extensions of the present analysis to more complex problems, involving kinetic coupling of diffusion, chemical reaction, and heat flux.

Within the framework of this type of analysis, the value of linear kinetic models [16] becomes apparent, as they apply exactly to departures from equilibrium in reaction boundary layers. There are, however, exceptions to this, as represented by the special case of "secondorder" boundary layers discussed above. In this regard, it should be emphasized that, as indicated by the examples considered above, the convergence of the "near-equilibrium" expansions will in general depend crucially on both the form of the reaction kinetics and the relative magnitudes of other parameters in the problem.

Acknowledgments - This work was partially supported by grants from the Public Health Service (GM-13402 and GM-15152 to J. S. Schultz). The authors are greatly indebted to H. Kutchai, J. A. Jacquez and F. J. Mathers, of the University of Michigan Department of Biostatistics, for permission to cite the numerical calculations from the manuscript of their forthcoming paper.

\section{NOTATION}

$A, B, C$ chemical species in reaction (2.1) (A is the transferred species)

$a, b$ stoichiometric ratios in (2.1)

C concentration variables, moles/vol.

$D$ molecular diffusivity, area/time 
$F \quad$ Friedlander-Keller factor in (3.2) and (3.3)

$f$ facilitation, Eq. (3.36)

$K$ dimensionless equilibrium constants in (3.6), (3.29), and (3.31)

$L$ membrane thickness, length

$N$ molar flux, moles/area-time

$r$ rate of reaction (2.1), production of carrier C, moles/vol-time

$S$ dimensionless flux ratio (or Sherwood number) of (2.14)

$x$ distance coordinate through the membrane, length

$y=x / L$ dimensionless distance

$\bar{y}, \underline{y}$ strained coordinates defined in (2.23) and (2.26)

Greek symbols

$\alpha, \beta$ constants in the kinetic constraints (2.11)

$\Delta$ boundary-layer parameters defined in (2.28), (2.29), and (2.31)

$\epsilon$ perturbation parameter of (2.9)

$\lambda$ boundary-layer parameters defined in (2.36) and (2.37)

$\sigma$ parameter defined by (3.31)

$\phi$ dimensionless kinetic function, defined in (2.9)

$\phi^{i} \quad \partial \phi / \partial \psi_{i}$

$\phi^{i j} \quad 1 / 2\left(\partial^{2} \phi / \partial \psi_{i} \partial \psi_{j}\right)$
$\Phi, \Phi^{i}, \Phi^{i j}$ the values of $\phi, \phi^{i}, \phi^{i j}$ evaluated at $\psi_{i}=\Psi_{i}^{\left({ }^{(\theta)}\right.}$

$\psi$ dimensionless concentrations, defined in (2.9)

$\Psi$ core expansion coefficients in (2.15)

Subscripts

$i, j k, \ldots(A, B, C)$ refer to the species
$1,2,3, \ldots(\mathbf{A}, \mathbf{B}, \mathbf{C})$ as in $(2.9)$

Superscripts

such as $p$ etc., in parentheses on symbols such as $\psi^{(p)}$, etc., refer to the order $p=0,1,2, \ldots$ of term in perturbation series like (2.15), (2.16) and (2.22)

$i, j, k$ on $\phi^{i}, \phi^{j}$, etc., refer to derivatives, as above. (The summation convention for repeated indices is explained immediately after (2.17))

\section{Overbars}

on quantities like $C, \psi, \phi, \lambda$ refer in general to left-hand $(y=0)$ boundary values or expansions, and

\section{Underbars}

refer to the corresponding right-hand $(y=1)$ values

\section{REFERENCES}

[1] ACRIVOS A., Chem. Engng Education, Am. Soc. Engng Education 1968262.

[2] BASSETT R. J., Ph.D. Dissertation, University of Michigan 1969. Excerpts to appear.

[3] BATCHELOR G. K.,J. Fluid Mech. 19561177.

[4] BROKAW R. S., J. chem. Phys. 1961351569.

[5] COFFIN K. P., J. chem. Phys. 1959311290.

[6] DeGROOT S. R. and MAZUR P., Non-Equilibrium Thermodynamics. North Holland 1962.

[7] FATT I. and LaFORCE R. C., Nature, Lond. 1962195505.

[8] FRIEDLANDER S. K. and KELLER K. H., Chem. Engng Sci. 196520121.

[9] FRIEDLANDER S. K. and KELLER K. H., Chem. Engng Prog. Symp. Ser. 19666289.

[10] GODDARD J. D. and ACRIVOS A.,J. Fluid Mech. 196624339.

[11] Handbook of Mathematical Functions (Edited by M. ABRAMOWITZ and 1. A. STEGUN). U.S. Dept. of Commerce, Nat'l Bureau of Standards, Applied Mathematics, Series 55, 1965.

[12] HEMMINGSEN E. A., Acta physiol. scand. Suppl. 1965246.

[13] HIRSCHFELDER J. O.,J. chem. Phys. 195926274.

[14] MURRAY J. D., Math. Biosci. 19682379.

[15] OLANDER D. L., A.I.Ch.E.Jl 19606233.

[16] ULANOWICZ R. E. and FRAZIER G. C. Jr., Chem. Engng Sci. 1968231335.

[17] VAN DYKE M., Perturbation Methods in Fluid Mechanics. Academic Press 1964.

[18] WARD W. J. and ROBB W. L., Science 19671561481. 
[19] WATSON G. N. Theory of Bessel Functions. Cambridge University Press 1922.

[20] WYMAN J., J. biol. Chem. 1966241115.

[21] ZILVERSMIT D. B., Science 1965149874.

\section{APPENDIX GENERAL SOLUTIONS \\ FOR THE BOUNDARY-LAYERS}

Here we present a brief outline of the general solution for the boundary-layer expansion coefficients $\bar{\psi}_{i}^{(p)}(\bar{y})$ in $(2.22)$, which is equally applicable to both the right-hand and left-hand boundary-layer expansions.

For any given order of the perturbation, $p=1,2, \ldots$ the Eqs. (2.24), (2.25), and (2.28) have the following general form:

$$
\frac{\mathrm{d}^{2} \psi_{i}^{(p)}}{\mathrm{d} y^{2}}-\mu_{i} \phi^{i} \psi_{j}^{(p)}=\sigma_{i}^{(p)}(y)
$$

with

$$
\psi_{1}{ }^{(p)}=\frac{\mathrm{d} \psi_{2}{ }^{(p)}}{\mathrm{d} y}=\frac{\mathrm{d} \psi_{3}{ }^{(p)}}{\mathrm{d} y}=0, \quad \text { at } y=0,
$$

and

$$
\psi_{i}^{(p)} \rightarrow \theta_{i}^{(p)}(y), \text { for } y \rightarrow \infty,
$$

where we have simply dropped the underbars and overbars on $\psi, y$ et cetera, which would otherwise distinguish between the right-hand and left-hand boundaries. In (A.1) the functions $\sigma_{i}^{(p)}(y)$ represent "non-homogeneous" terms of the type appearing in the differential Eqs. (2.24), involving presumably known, lower-order perturbations, while the $\theta_{i}^{(p)}(y)$ are polynomials of degree $p$, of the kind occurring either in (2.28) or in (2.29).

By standard techniques of linear algebra one can solve (A.1), most conveniently in terms of the difference $\psi_{i}^{(p)}-\theta_{i}^{(p)}$, to yield formally

$$
\begin{aligned}
\psi_{i}^{(p)}(y)= & \int_{0}^{y}\left[\left(\delta_{i}{ }^{j}-\frac{\mu_{i} \phi^{j}}{\lambda^{2}}\right)\left(y-y^{*}\right)+\frac{\mu_{i} \phi^{j}}{\lambda^{2}} \sinh \lambda\left(y-y^{*}\right)\right] \\
& \times\left[\sigma_{j}^{(p)}\left(y^{*}\right)-\frac{\mathrm{d}^{2} \Theta_{j}^{(p)}}{\mathrm{d} y^{2}}\left(y^{*}\right)+\mu_{j} \phi^{k} \Theta_{k}^{(p)}\left(y^{*}\right)\right] \mathrm{d} y^{*} \\
& +\Theta_{i}^{(p)}(y)+\mu_{i} \xi^{(p)} \mathrm{e}^{-\lambda y}+\mu_{i} \eta^{(p)} \mathrm{e}^{+\lambda y}
\end{aligned}
$$

where

$$
\lambda^{2}=\phi^{j} \mu_{j}(>0),
$$

$\xi^{(p)}, \eta^{(p)}$ are constants, and the symbol $\delta_{i}{ }^{j}$ denotes a "Kronecker delta" $\left(\delta_{i}{ }^{j}=1\right.$ for $i=j, \delta_{i}{ }^{j}=0$ otherwise, and, in accordance with our textual convention all the terms in (A.1-3) that involve the repeated indices $j, k$ are to be summed over $j, k=1,2,3$ ).

The constants $\xi^{(p)}$ and $\eta^{(p)}$ must be chosen to satisfy the side conditions at $y=0$ and $y=\infty$ in (A.1). In particular, since both the integral in (A.2) and its first $y$-derivative vanish at $y=0$, the boundary conditions at $y=0$ give

$$
\Psi_{1}^{(p)}(0)+\mu_{1}\left(\xi^{(p)}+\eta^{(p)}\right)=0
$$

and

$$
\frac{\mathrm{d} \Psi_{i}^{(p-1)}}{\mathrm{d} y}(0)+\lambda \mu_{i}\left(\eta^{(p)}-\xi^{(p)}\right)=0, \text { for } i=2,3 \text {, }
$$

where we have made use of the fact that the values of the polynomial $\Theta_{i}^{(p)}$ and its $y$-derivative at $y=0$ are equal to those of the core functions $\Psi_{i}^{(p)}, \Psi_{t}^{(p-1)}$, respectively. (The equations have been written for the left boundary only). Also, one will note that the last equation in (A.4) is compatible with the integral relation of (2.21).

Now we observe that, at a given order $p$ of the perturbation, the quantity $\Psi_{i}^{(p)}(0)$ in the first equation of (A.4) is unknown. Indeed, it must be determined from the boundarylayer calculation at hand, which is accomplished via the last condition in (A.1), at $y=\infty$. This latter step requires, however, some further consideration of the integral in (A.2).

First of all we note that the second factor in brackets [ ] in the integrand of (A.2) will not involve any residual algebraic or constant terms for $y^{*} \rightarrow \infty$, but, rather, only exponentially small terms, $\mathrm{e}^{-\lambda y^{*}}, y^{*} \mathrm{e}^{-\lambda y^{*}}, \ldots, \mathrm{e}^{-2 \lambda y^{*}}, \ldots$ For the loworder perturbations considered here, $p=1,2$, this can be verified directly by inspection of the non-homogeneous terms in $(2.24)\left(\sigma_{i}^{(p)}\right)$ and by means of the definition of $\Theta_{i}^{(p)}$, the polynomials in (2.28) or (2.29). Thus, one finds that the coefficients of any purely algebraic terms of the type $\left(y^{*}\right)^{n}$, $n=0,1,2, \ldots$, which appear in the integrand of (A.2), can be identified directly with the core Eqs. (2.18)-(2.20) or the higher $y$-derivatives of these equations evaluated at $y=0$; in other words, with the values of

$$
\Phi, \frac{\mathrm{d} \Phi}{\mathrm{d} y}, \frac{\mathrm{d} \Phi}{\mathrm{d} y^{2}}, \ldots, \Phi^{k} \Psi_{k}, \frac{\mathrm{d}}{\mathrm{d} y} \Phi^{k} \Psi_{k}, \ldots,
$$

at $y=0$, which are identically zero by $(2.18)-(2.20)$. (Although the same kind of result would doubtless hold for higher-order terms $p=3,4 \ldots$, we have not actually gone to the lengths of a rigorous verification for terms beyond those of direct concern here).

By means of the considerations of the preceding paragraph one can show readily that, as it stands, the function $\psi_{i}^{(p)}$ in (A.2) behaves like

$$
\begin{aligned}
\psi_{i}^{(p)}-\Theta_{i}^{(p)} & +\mu_{i} \mathrm{e}^{\lambda y}\left\{\eta^{(p)}-\frac{\phi^{3}}{2 \lambda^{3}} \int_{0}^{\infty} \mathrm{e}^{-\lambda y^{*}}\left[\sigma_{j}^{(p)}\left(y^{*}\right)\right.\right. \\
& \left.\left.-\frac{\mathrm{d}^{2} \Theta_{j}^{(p)}}{\mathrm{d} y^{2}}\left(y^{*}\right)+\mu_{j} \phi^{k} \Theta_{k}^{(p)}\left(y^{*}\right)\right] \mathrm{d} y^{*}\right\}, \text { for } y \rightarrow \infty .
\end{aligned}
$$

Hence, to satisfy the condition at $y=\infty$ of (A.1), we must have

$$
\begin{aligned}
& \eta^{(p)}=\frac{\phi^{j}}{2 \lambda^{3}} \int_{0}^{\infty} \mathrm{e}^{-\lambda y^{*}}\left[\sigma_{j}^{(p)}\left(y^{*}\right)-\frac{\mathrm{d}^{2} \Theta_{j}^{(p)}}{\mathrm{d} y^{2}}\left(y^{*}\right)\right. \\
& \left.+\mu, \phi^{k} \Theta_{k}^{(p)}\left(y^{*}\right)\right] \mathrm{d} y^{*} .
\end{aligned}
$$

Equations (A.5) and (A.4) suffice to determine completely the constants $\xi^{(p)}, \eta^{(p)}$, as well as the boundary-value at $y=0$ for the core-expansion function, $\Psi^{(p)}(0)$. By similar considerations one can also obtain the right-hand boundary value $\Psi^{(p)}(1)$

To summarize, then, the boundary-layer analysis at any order $p=1,2, \ldots$ gives us the boundary-layer coefficients 
$\bar{\psi}_{i}^{(p)}(\bar{y})$ in $(2.22)$, in addition to the boundary value $\Psi_{1}^{(p)}(0)$ on the $p$ th core-expansion coefficient $\Psi_{1}{ }^{(p)}(y)$ at $y=0$ (as well as $\Psi_{i}^{(p)}(y)$ and $\Psi_{1}^{(p)}(1)$, at the right-hand boundary). In this connection, and as a final remark, we note that the flux of the transferred species is determined to terms $0\left(\epsilon^{p-1}\right)$ relative to the leading term by the derivative (at the left boundary, say).

$\frac{\mathrm{d} \bar{\psi}_{1}^{(p)}}{\mathrm{d} \bar{y}^{1}}(0)=\frac{\mathrm{d} \Psi_{1}^{(p-1)}}{\mathrm{d} y}(0)+\lambda \mu_{1}\left(\eta^{(p)}-\xi^{(p)}\right)$

$$
\begin{aligned}
& =\frac{\mathrm{d} \Psi_{1}^{(p-1)}}{\mathrm{d} y}(0)-\frac{\mu_{1}}{\mu_{2}} \frac{\mathrm{d} \Psi_{2}^{(p-1)}}{\mathrm{d} y}(0) \\
& =\frac{\mathrm{d} \Psi_{1}^{(p-1)}}{\mathrm{d} y}(0)-\frac{\mu_{1}}{\mu_{3}} \frac{\mathrm{d} \Psi_{3}^{(p-1)}}{\mathrm{d} y}(0),
\end{aligned}
$$

the last two equalities following from (A.4).

Résumé - Ici on présente une analyse du problème de l'augmentation (〈(facilitation $\rangle)$ de la diffusion massique permanente, à travers une membrane d'eppaiseur finie, grâce à la présence d'une réaction chimique, homogène, rapide, et réversible. Dans le cas limite d'une réaction infiniment rapide, on se sert de la méthode dite (〈method of matched asymptotic expansions〉) pour mettre en évidence la structure asymptote du champs de diffusion, lequel on demontre est composé d'une région centrale (un $\langle\langle$ noyau $\rangle\rangle)$ d'équilibre bornée par des couches limites de réaction situées aux deux extrémités de la membrane. Et alors par cette méthode on developpe, jusqu'au terme du deuxième ordre, une séries d'approximations successives pour représenter l'éffet de l'écart d'equilibre sur le flux massique. Plusieurs exemples, parmi lesquels le système oxygène-hémoglobine, sont traités par la méthode, et des comparaisons sont faits avec d'autres solutions, analytiques aussi bien que numériques.

Zusammenfassung - Es wird über eine Analyse des "geförderten" Transportproblems berichtet, in welchem stetige Diffusion durch eine begrenzte Membran, begleitet von einer rapiden und reversiblen, homogenen chemischen Reaktion stattfindet. Als Grenz unendlich schneller Reaktionen wurde das Verfahren der angepassten asymptotischen Expansionen verwendet, um die asymptotische Gestaltung des Diffusionsfeldes zu analysieren, welche sich als aus einem "Gleichgewichtskern" zusammen mit "Grenzschicht" Reaktionzonen an den Membrangrenzen bestehend erweist. Es werden asymptotische Expansionen abgeleitet, die es grundsätzlich ermöglichen sollten die Wirkungen sowohl erster als auch zweiter Ordnung einer Abweichung vom Reaktionsgleichgewicht auf die Restgeschwindigkeiten der Stoffübertragung durch Diffusion zu berechnen. Es werden verschiedene Beispiele erörtert, von welchen eines ein Modell der Sauerstoffdiffusion durch Hämoglobinlösungen darstellt, und es werden Vergleiche zweichen analytischen und numerischen Lösungen angegeben. 